\title{
EDITORIAL
}

\section{An ESCP message}

\section{J. W. F. van Mil}

Received: 18 October 2010/Accepted: 25 October 2010/Published online: 13 January 2011

(C) Springer Science+Business Media B.V. 2010

The International Journal for Clinical Pharmacy is supported by the ESCP, the European Society of Clinical Pharmacy. ESCP members have free access to the journal and the archives, dating back to 1979, when the journal was still called Pharmaceutisch Weekblad Scientific Edition (later PWS, or Pharmacy World \& Science).

Since some time ESCP communicates with its members through email. It is noted, however, that some emails do not arrive at their destinations. This is usually due to spamfilters that do not allow bulk-messages through.
If you know that your institution (or your own computer) works with a spam filter, make sure it allows you to receive messages from escp@planet.nl, escp@bluewin.ch, mci-group.com, or escpweb.org, and add the addresses or domains to the positive list(s)!

Please also note that the membership can be renewed on-line (see www.escpweb.org), and that is the easiest option for both the members as well as the office.

On behalf of the European Society of Clinical Pharmacy (ESCP)

J. W. F. van Mil $(\bowtie)$

Margrietlaan 1, 9471 CT Zuidlaren, The Netherlands

e-mail: jwfvmil@planet.nl 